\title{
Studying Ultrafast Spectral Diffusion and Correlation Dynamics using Two-Dimensional Electronic Spectroscopy
}

\author{
Thanh Nhut Do ${ }^{1}$, Hoang Long Nguyen ${ }^{1}$, Jamie H. N. Sim ${ }^{1}$, Xuan Leng ${ }^{1}$, Duc Viet Le ${ }^{1}$, M. \\ Faisal Khyasudeen ${ }^{1,2}$, Pawel J. Nowakowski ${ }^{1}$, and Howe-Siang Tan ${ }^{\mathbf{1}}$ \\ ${ }^{1}$ Division of Chemistry and Biological Chemistry, School of Physical and Mathematical Sciences, \\ Nanyang Technological University, 21 Nanyang Link, Singapore 637371, Singapore \\ ${ }^{2}$ Department of Chemistry, Faculty of Science, University of Malaya, 50603 Kuala Lumpur, \\ Malaysia \\ E-mail: howesiang@ntu.edu.sg
}

\begin{abstract}
The Frequency Fluctuation Correlation Function (FFCF) is a measure of the spectral diffusion dynamics of an electronic transition and details the interaction between the chromophore and its environment. The Frequency Fluctuation Cross-Correlation Function (FXCF) allows us to study how correlated, the transitions between different electronic transitions, are. These quantities contain a wealth of information on how the electronic transitions in chromophores and excitonic states interact and couple with their environment, and with each other. We summarize the experimental implementations and theoretical considerations of using ultrafast coherent two-dimensional electronic spectroscopy (2DES) to characterize FFCFs and FXCFs. In 2DES, the measured peakshapes of the electronic transitions are highly sensitive to the spectral diffusion and correlation dynamics [1]. One method that allows us to analyse these peakshapes is the Centre Line Slope (CLS) method [2]. We describe applications to systems such as the chlorophyll (Chl) molecules which are the major pigment molecules in the plant photosynthetic light harvesting machinery. We characterize the FFCFs of the $\mathrm{Q}_{\mathrm{y}}$ transition of $\mathrm{Chl} \mathrm{a}$ and $\mathrm{Chl} \mathrm{b}$ in various solvent environment $[3,4]$. The solvent dependence of the FFCF values and the relation of these values to the molecule's interaction with the solvent environment, will be discussed. Using similar methods, the FXCF between the $\mathrm{Q}_{\mathrm{x}}$ and $\mathrm{Q}_{\mathrm{y}}$ transitions of Chl a are measured. The results indicate that the $\mathrm{Q}_{\mathrm{x}}$ and $\mathrm{Q}_{\mathrm{y}}$ transitions have only minimal initial correlation, which decays to zero in a timescale of about 2 ps [5]. We also characterize the FFCFs of CdSe quantum dots (QD) and nanoplatelet (NPL) [6]. We found that no spectral diffusion dynamics occurs for the CdSe QDs. On the other hand, spectral diffusion was observed in the CdSe 5 mono-layers NPLs heavy-hole transition. The normalized Frequency Fluctuation Correlation Function (FFCF) of the CdSe NPLs heavy-hole transition was measured to have a major fast decay component at $<200 \mathrm{fs}$.
\end{abstract}

Keywords: Ultrafast Spectroscopy, Two-dimensional Electronic Spectroscopy, Spectral Diffusion, Chlorophyll, Nanoplatelets.

\section{References:}

[1] F.D. Fuller, J.P. Ogilvie, Annu. Rev. Phys. Chem. 2015, 66, 667-690.

[2] K. Kwak, S. Park, I.J. Finkelstein, and M.D. Fayer, J. Chem. Phys. 2007, 127, 124503.

[3] Wells K.L.; Zhang Z.; Rouxel J.R.; Tan H.-S. J. Phys. Chem. B 2013, 117 (8), 2294-2299.

[4] M. Faisal Khyasudeen, P.J. Nowakowski, H.L. Nguyen, J.H.N. Sim, T.N. Do, and H.-S. Tan, Chem. Phys. 2019, 527, 110480.

[5] Faisal Khyasudeen M.; Nowakowski P.J.; Tan H.-S. J. Phys. Chem. B 2019, 123 (6), 1359-1364.

[6] T.N. Do, C. Zhang, X. Ong, J, Lian, Y. Chan, H.-S. Tan, MRS Advances 2019, 4, (1), 1-7. 